\title{
Simulação como estratégia de ensino em enfermagem:
}

\author{
revisão de literatura
}

Ilka Nicéia D'Aquino Oliveira Teixeira ${ }^{1}$

Jorge Vinícius Cestari Felix ${ }^{2}$

TEIXEIRA, I.N.D.O.; FELIX, J.V.C. Simulation as a teaching strategy in nursing education: literature review. Interface - Comunic., Saude, Educ., v.15, n.39, p.1173-83, out./dez. 2011.

The objective of this systematic review is to present simulation as a teaching strategy for undergraduate nursing students, as described in Brazilian studies. The search was carried out in the databases BDENF, Lilacs and Medline, using the following keywords: teaching, education, laboratory, simulation, nursing. The included studies were the scientific papers on simulation as a teaching strategy for undergraduate nursing students, published in Portuguese, between January 1999 and September 2010. Bardin's thematic content analysis was applied to data analysis. Nine papers met the inclusion criteria, being classified into two categories: seven in "computer simulation", and two in "nursing laboratory". The results indicate that simulation encourages both the acquisition of psychomotor skills and self-confidence. Yet, no clinical trial was found on the efficacy of simulation for improving nursing students' practice. Thus, there is a need for further research on the subject.

Keywords: Computer-assisted instruction. Nursing students. Simulation.
O objetivo desta revisão sistemática é apresentar a simulação como estratégia de ensino para estudantes de graduação em enfermagem, conforme estudos brasileiros. A busca foi realizada nas bases de dados BDENF, Lilacs e Medline, utilizando-se as palavras-chave: ensino, educação, laboratório, simulação e enfermagem. Foram incluídos os artigos científicos sobre simulação como estratégia de ensino para estudantes de enfermagem publicados em português, de janeiro de 1999 a setembro de 2010. Para análise dos dados, os autores aplicaram a técnica de análise temática de conteúdo, descrita por Bardin. Nove artigos foram selecionados, sendo classificados em duas categorias: sete em "Simulação por Computador" e dois em "Laboratório de Enfermagem". Os resultados indicam que a simulação contribui para a aquisição de habilidades psicomotoras e autoconfiança. Não foram encontrados ensaios clínicos sobre a eficácia da simulação na prática dos estudantes de enfermagem. Há necessidade de mais pesquisas sobre o tema.

Palavras-chave: Instrução por computador. Estudantes de Enfermagem. Simulação.

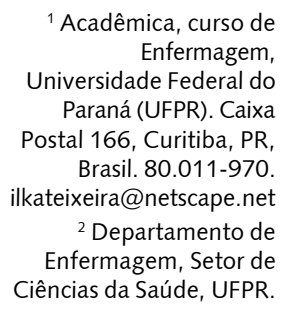

${ }^{1}$ Acadêmica, curso de Enfermagem, Universidade Federal do Paraná (UFPR). Caixa Postal 166, Curitiba, PR, Brasil. 80.011-970.

ilkateixeira@netscape.net

${ }^{2}$ Departamento de Enfermagem, Setor de Ciências da Saúde, UFPR. 


\section{Introdução}

Nas aulas práticas, os cuidados aos pacientes são experiências que geram ansiedade nos estudantes de enfermagem. Para alguns acadêmicos, a clínica é o período mais estressante do curso de graduação, e os seguintes fatores estão relacionados à angústia: inexperiência, medo de cometer erros e condições de avaliação (Beck, Srivastava, 1999). Hart e Rotam (1994) avaliaram o nível de estresse de 94 estudantes de enfermagem, reafirmando as dificuldades emocionais encontradas nas atividades clínicas devido à insegurança na realização dos procedimentos e ao despreparo na execução das técnicas.

Os problemas na prática dos alunos são fatores frequentes na educação em enfermagem, e estão relacionados à ansiedade durante a supervisão e a avaliação (Sharif, Masoumi, 2005). Alguns estudantes referem distúrbios intestinais e urinários durante as aulas práticas; outros recusam-se a realizar os procedimentos (Gomes, Germano, 2007). Essas reações são constatadas mesmo em situações simuladas no laboratório de enfermagem, sobretudo na realização de procedimentos invasivos.

Os professores precisam selecionar as oportunidades de intervenções para a prática do cuidado, pois os acadêmicos são diferentes em idade, estilo de vida, experiências e talentos. Alguns estudantes estão tendo o primeiro contato com as tecnologias de curativos e a administração de medicamentos, enquanto outros são profissionais da área. A reflexão dos docentes sobre o desempenho próprio torna efetiva a transmissão das técnicas requeridas para os procedimentos, sendo considerada uma estratégia educacional (Jeffries, 2005).

Outra estratégia é a simulação, que está inserida no currículo de enfermagem para o ensino de técnicas e procedimentos clínicos, incluindo diferentes objetos: jogos, modelos anatômicos, manequins, bonecos, estudos de casos e apresentações de multimídia (Tuoriniemi, Schott-Bauer, 2008). As simulações que utilizam manequins facilitam a aquisição de habilidades para os cuidados aos pacientes, propiciando a imersão dos estudantes em ambientes interativos seguros para desenvolver o processo de enfermagem (Gomes, Germano, 2007).

Considerando que a segurança do paciente é imprescindível, inicialmente, os acadêmicos participam de aulas teóricas e práticas, com simulações no laboratório de enfermagem; posteriormente, desenvolvem os primeiros cuidados em instituições de saúde (Gomes, Germano, 2007). O laboratório de enfermagem é um recurso com estrutura para a aprendizagem, que dispõe de equipamentos e materiais simuladores para o desenvolvimento de habilidades profissionais, tais como: avaliação do paciente, desempenho psicomotor, pensamento crítico para a solução de problemas e colaboração interdisciplinar (Rothgeb, 2008; Coutinho, Friedlander, 2004).

Os cenários de simulação oferecem experiências cognitivas, psicomotoras e afetivas, contribuindo para a transferência de conhecimento da sala de aula para os ambientes clínicos (Tuoriniemi, SchottBauer, 2008). Nessas experiências, os docentes devem reforçar os acertos nos procedimentos, corrigir os erros e explicar os pontos nos quais há necessidade de aprimoramento dos acadêmicos (Rothgeb, 2008).

As simulações computacionais contribuem para a qualidade dos cuidados oferecidos aos pacientes. Há programas educativos e jogos online que permitem o treinamento de procedimentos clínicos em situações de simulação, sendo frequente o desenvolvimento de objetos digitais para esse fim (Schatkoski et al., 2007; Melo, Damasceno, 2006; Barbosa, Marin, 2000). É importante, no entanto, que a produção de software para a educação não se caracterize pelo reducionismo tecnológico, com excessiva preocupação com o design, em detrimento da finalidade pedagógica (Telles Filho, Cassiani, 1999).

As ferramentas computacionais oferecem subsídios para o processo de enfermagem, com materiais educacionais que incluem arquivos de áudio e vídeo (Barbosa, Marin, 2000). A informática é um recurso em potencial para a aprendizagem de técnicas e procedimentos de risco, sendo recomendada a inserção dessa disciplina nos currículos dos cursos de bacharelado em Enfermagem (Dias et al., 2005).

As práticas com simulações devem ser planejadas em uma sequência de complexidade crescente, conforme as exigências das disciplinas, possibilitando aos estudantes demonstrarem competência em cada nível do curso de Enfermagem (Medley, Horne, 2005; Nehring, Lashley, 2004). Os simuladores podem ser caracterizados pelo grau de fidelidade, como: baixa fidelidade, exemplificada por manequins 
estáticos; média, permitindo alguma proximidade com a realidade; e alta fidelidade (Jeffries, 2007). Os simuladores de alta fidelidade representam o corpo humano, com semelhanças na aparência, sentimentos e respostas aos cuidados, inclusive ao oxigênio e às medicações, contribuindo para estimular o pensamento clínico (Seropian et al., 2004). Esses simuladores podem ser programados para responder às falhas na administração de medicamentos e na performance de habilidades, demonstrando, aos estudantes, as possíveis consequências dos erros (Rothgeb, 2008).

A experiência de simulação promove o pensamento crítico dos estudantes, contemplando cinco fatores: objetivos, fidelidade, solução do problema, apoio e feedback (Jeffries, 2007). Os objetivos indicam as orientações para a aprendizagem. A fidelidade é o parâmetro de aproximação da realidade, cujo ambiente apresenta características específicas do cenário: clínica, quarto de hospital, enfermaria, ambulatório ou domicílio. Os papéis dos estudantes são definidos previamente à simulação, e o caso clínico do "paciente" deve ser um desafio com solução possível (Rothgeb, 2008). Os manequins são vestidos como seres humanos e podem ter lesões, feridas, incisões e drenos, sendo comandados por controle remoto e programados para perguntar e responder questões específicas. As orientações docentes podem ser sutis, permitindo que o estudante seja responsável pela tomada de decisões no processo (Rothgeb, 2008). O feedback deve acontecer imediatamente após a simulação, observando os princípios de adequação, pontualidade, frequência e interação (Beckman, Lee, 2009).

A evolução dos estudantes em direção à competência resulta da participação ativa nas simulações, das observações de experiências dos colegas e do feedback dos docentes (Seropian et al., 2004). A autoavaliação também é importante; mas, quando o acadêmico reconhece a própria atuação como não satisfatória, o(a) professor(a) o orientará sobre as ações possíveis para melhorar o desempenho.

As simulações humanizam o ensino e contribuem para a superação das dificuldades e para o controle do estresse emocional dos acadêmicos, reforçando a relevância da interação entre os professores e os estudantes (Gomes, Germano, 2007). A expectativa é que, a partir das experiências de simulação, haja uma redução de erros nos procedimentos em situações clínicas, em um continuum de ação e reflexão no processo de enfermagem.

Os enfermeiros docentes do século 21 devem buscar estratégias inovadoras para o ensino da prática, sendo a simulação uma ferramenta efetiva na educação e no contexto moderno do cuidado à saúde (Rothgeb, 2008). No Brasil, as pesquisas sobre simulação estão na fase inicial; mas os docentes e os discentes têm demonstrado atitudes favoráveis ao uso dessa estratégia para o ensino de procedimentos e técnicas de enfermagem (Barbosa, Marin, 2000). Vários estudos sugerem que a tecnologia emergente da simulação clínica aproxima o treinamento dos procedimentos reais; mas, é necessário investigar se há evidências científicas sobre o efeito positivo das simulações na educação (Gomes, Germano, 2007; Melo, Damasceno, 2006; Barbosa, Marin, 2000). Dessa forma, duas questões orientam esta revisão da literatura: (1) Quais são os estudos brasileiros sobre o uso de simulações para o ensino de procedimentos e técnicas em enfermagem? (2) Há evidências científicas de que as simulações exercem efeito positivo na aprendizagem dos acadêmicos de graduação em enfermagem?

O objetivo deste estudo é apresentar o uso de simulações no ensino de procedimentos e técnicas para os estudantes de graduação de enfermagem, conforme os artigos científicos publicados em periódicos do Brasil, no período de janeiro de 1999 a setembro de 2010.

\section{Percurso metodológico}

Este é um estudo descritivo de revisão sistemática da literatura sobre o uso de simulações, como fatores contribuintes para o ensino de técnicas e procedimentos, no curso de graduação em Enfermagem. A pesquisa consistiu na busca de artigos científicos sobre o tema, publicados em periódicos nacionais, na língua portuguesa, no período de janeiro de 1999 a setembro de 2010. Os autores consultaram as bases de dados BDENF, Lilacs e Medline, sendo os parâmetros de busca e limites definidos segundo as opções disponíveis em cada base. Na Medline, aplicaram-se os termos da MeSH, (learning OR education) AND (laboratory OR simulation) AND nursing, observando os limites: humans, nursing journal articles, Portuguese. Para a busca na BDENF e Lilacs, foi utilizado o sistema da Biblioteca 
Virtual em Saúde, com os termos (ensino OR educação) AND (laboratório OR simulação) AND enfermagem, observando-se os mesmos limites da Medline.

A seleção da literatura seguiu três critérios de inclusão previamente estabelecidos: (1) artigo científico, com o objetivo diretamente relacionado ao uso de simulações no ensino de técnicas e procedimentos para acadêmicos de graduação em enfermagem; (2) estar publicado em periódico nacional no idioma português; (3) publicação no período de janeiro de 1999 a setembro de 2010. Dessa forma, para o presente estudo, os autores excluíram os livros, as dissertações e as teses.

Inicialmente, foi realizada uma busca automática, atentando para os parâmetros supracitados. A seguir, houve uma seleção dos estudos em três etapas: (1) exclusão de artigos não pertinentes ao objetivo da presente pesquisa; (2) conferência dos títulos dos trabalhos e dos respectivos autores para se verificarem repetições em mais de uma base de dados, sendo os artigos redundantes computados apenas uma vez; (3) leitura dos resumos, com análise da relação entre os objetivos de cada estudo e o propósito desta pesquisa, para exclusão dos artigos não pertinentes. Cumpridas as três etapas descritas, procedeu-se à leitura, na íntegra, dos artigos selecionados.

Na sequência, os autores aplicaram a análise de conteúdo para tratamento dos dados, adaptada da técnica de Bardin (2004). Segundo Caregnato e Mutti (2006), a análise de conteúdo pode ser realizada em textos, que são originados de transcrições de entrevistas, e em textos já existentes, como é o caso dos artigos selecionados para este estudo. Assim, essa técnica é indicada quando os dados apresentamse sob a forma de textos, e tem sido utilizada para análise em pesquisas qualitativas, compreendendo três etapas: (1) pré-análise, na qual é feita a leitura flutuante para a elaboração de hipóteses e de indicadores que fundamentam a interpretação dos dados; (2) exploração do material para a codificação dos dados, a partir das unidades de registros; (3) tratamento dos dados e alcance de inferências, que consiste na classificação dos elementos explorados, considerando-se as semelhanças e as diferenças entre eles, para posterior categorização com ênfase nas características comuns.

Neste trabalho, a análise de conteúdo resultou nas duas categorias para a classificação dos artigos selecionados: Simulação por Computador e Laboratório de Enfermagem.

\section{Resultados e discussão}

A busca automática gerou 25 estudos, apresentando oito na BDENF, 12 na Lilacs e cinco na Medline. Seis trabalhos estavam repetidos, sendo dois indexados nas três bases de dados consultadas e quatro contidos na BDENF e na Lilacs. Os artigos repetidos foram contados apenas uma vez, totalizando 17 estudos. Após a aplicação dos critérios estabelecidos, foram excluídos oito artigos com as seguintes justificativas: não-disponibilidade da publicação na íntegra (um); realização do estudo na cidade do México (um); educação continuada para enfermeiros gerentes (um); aplicação do modelo de educação de laboratório para ensino de técnicas de gestão em enfermagem (dois); elaboração de material educativo sem uso de simulação (um); relato de experiência para aprimoramento de monitores (um); conteúdo teórico sobre sistema de informação aplicado à enfermagem (um).

De 1999 a 2003, apenas um estudo estava indexado; mas, no período de 2004 a 2010, oito artigos constaram nas bases de dados. Os estudos foram divulgados em periódicos, em quantidade indicada conforme os números entre parênteses: Revista da Escola de Enfermagem da USP (dois); Revista Gaúcha de Enfermagem (dois); Revista Latino Americana de Enfermagem (dois); Acta Paulista de Enfermagem (um); Texto e Contexto em Enfermagem (um) e Online Brazilian Journal of Nursing (um).

Portanto, nesta revisão de literatura, foram incluídos nove artigos científicos sobre o uso de simulações para o ensino de técnicas e procedimentos para estudantes de graduação em enfermagem. Conforme o conteúdo, os estudos selecionados foram classificados em duas categorias, sendo sete artigos sobre Simulação por Computador e dois classificados em Laboratório de Enfermagem (Quadros 1 e 2).

As amostras das pesquisas foram constituídas por acadêmicos do curso de graduação em enfermagem, com três exceções: uma amostra formada por docentes, em uma pesquisa que avaliou software para ensino de oxigenoterapia; um relato de experiência sobre a construção de software de 
Quadro 1. Artigos da categoria Simulação por Computador

\begin{tabular}{|c|c|c|c|c|c|}
\hline Autores & Objetivo & Amostra & Estudo/ Método & Resultados & Considerações \\
\hline $\begin{array}{l}\text { Schatkoski } \\
\text { et al. (2007) }\end{array}$ & $\begin{array}{l}\text { Avaliar objetos } \\
\text { educacionais } \\
\text { (oxigenoterapia) }\end{array}$ & 44 estudantes & $\begin{array}{l}\text { Quantitativo. } \\
\text { Questionário: avaliação } \\
\text { do material digital. }\end{array}$ & $\begin{array}{l}\text { Favoráveis aos } \\
\text { objetos } \\
\text { educacionais. }\end{array}$ & $\begin{array}{l}\text { Reforço à } \\
\text { participação do } \\
\text { estudante. }\end{array}$ \\
\hline $\begin{array}{l}\text { Melo e } \\
\text { Damasceno } \\
(2006)\end{array}$ & $\begin{array}{l}\text { Desenvolver software } \\
\text { para ensino de } \\
\text { ausculta pulmonar }\end{array}$ & Não se aplica & $\begin{array}{l}\text { Descritivo. Software em } \\
6 \text { etapas. }\end{array}$ & $\begin{array}{l}\text { Software disponível } \\
\text { em kit. }\end{array}$ & $\begin{array}{l}\text { Bibliografia } \\
\text { escassa. Aplicação } \\
\text { depende de } \\
\text { parceria. }\end{array}$ \\
\hline $\begin{array}{l}\text { Barbosa e } \\
\text { Marin } \\
(2000)\end{array}$ & $\begin{array}{l}\text { Desenvolver e avaliar } \\
\text { simulação via web em } \\
\text { terapia intensiva }\end{array}$ & $\begin{array}{l}4 \text { webdesigners } \\
4 \text { docentes, } 4 \\
\text { enfermeiros } \\
25 \text { estudantes }\end{array}$ & $\begin{array}{l}\text { Pesquisa aplicada. } \\
\text { Ambiente desenvolvido } \\
\text { em } 6 \text { fases. }\end{array}$ & $\begin{array}{l}\text { Ergonomia, } \\
\text { pedagogia } \\
\text { adequadas. } \\
\text { Simulação longa. } \\
\text { Não há valores de } \\
\text { referência. }\end{array}$ & $\begin{array}{l}\text { Validade da } \\
\text { simulação web } \\
\text { em terapia } \\
\text { intensiva. }\end{array}$ \\
\hline $\begin{array}{l}\text { Telles Filho e } \\
\text { Cassiani } \\
(1999)\end{array}$ & $\begin{array}{l}\text { Comparar atitudes } \\
\text { de discentes de } 2 \\
\text { instituições sobre } \\
\text { ensino com } \\
\text { computador }\end{array}$ & 101 discentes & $\begin{array}{l}\text { Quantitativo, descritivo. } \\
\text { Elaboração, aplicação e } \\
\text { análise de escalas. }\end{array}$ & $\begin{array}{l}\text { Aprovação do } \\
\text { computador no } \\
\text { ensino de } \\
\text { enfermagem. }\end{array}$ & $\begin{array}{l}\text { Atitudes positivas } \\
\text { sobre o } \\
\text { computador no } \\
\text { ensino. }\end{array}$ \\
\hline $\begin{array}{l}\text { Sasso e } \\
\text { Souza } \\
(2006)\end{array}$ & $\begin{array}{l}\text { Descrever a } \\
\text { construção e avaliar } \\
\text { ambiente simulado } \\
\text { em RCP }\end{array}$ & 3 estudantes & $\begin{array}{l}\text { Quantitativo, descritivo. } \\
6 \text { etapas na construção, } \\
\text { e avaliação do } \\
\text { programa. }\end{array}$ & $\begin{array}{l}\text { Ambiente simulado } \\
\text { para ensino de RCP. }\end{array}$ & $\begin{array}{l}\text { Ambiente } \\
\text { permite o } \\
\text { cuidado virtual do } \\
\text { paciente em RCP. }\end{array}$ \\
\hline $\begin{array}{l}\text { Cogo et al. } \\
(2009)\end{array}$ & $\begin{array}{l}\text { Investigar a opinião } \\
\text { de docentes sobre } \\
\text { objetos digitais } \\
\text { (oxigenoterapia) }\end{array}$ & 10 docentes & $\begin{array}{l}\text { Transversal, exploratório. } \\
\text { Questionário para avaliar } \\
3 \text { objetos educacionais. }\end{array}$ & $\begin{array}{l}\text { Avaliação } \\
\text { satisfatória dos } \\
\text { objetos } \\
\text { educacionais digitais. }\end{array}$ & $\begin{array}{l}\text { Objetos } \\
\text { educacionais } \\
\text { capacitam alunos. }\end{array}$ \\
\hline $\begin{array}{l}\text { Silva e Cogo } \\
(2007)\end{array}$ & $\begin{array}{l}\text { Avaliar o desempenho } \\
\text { na punção venosa, } \\
\text { com } 3 \text { objetos digitais }\end{array}$ & 37 estudantes & $\begin{array}{l}\text { Exploratório, descritivo. } \\
\text { Observação de } 10 \\
\text { etapas no procedimento } \\
\text { e questionário. }\end{array}$ & $\begin{array}{l}\text { Material contribui } \\
\text { para aprendizado } \\
\text { (91,90\% dos } \\
\text { acadêmicos). }\end{array}$ & $\begin{array}{l}\text { Simulação digital } \\
\text { contribui para o } \\
\text { aprendizado. }\end{array}$ \\
\hline
\end{tabular}

Quadro 2. Artigos da categoria Laboratório de Enfermagem

\begin{tabular}{|l|l|l|l|l|l|}
\hline \multicolumn{1}{|c|}{ Autores } & \multicolumn{1}{|c|}{ Objetivo } & Amostra & Estudo/ Método & \multicolumn{1}{c|}{ Resultados } & \multicolumn{1}{c|}{ Considerações } \\
\hline $\begin{array}{l}\text { Gomes e } \\
\text { Germano } \\
(2007)\end{array}$ & $\begin{array}{l}\text { Identificar } \\
\text { contribuições do } \\
\text { laboratório no ensino }\end{array}$ & 26 estudantes & $\begin{array}{l}\text { Qualitativo, } \\
\text { descritivo. } \\
\text { grupos focais }\end{array}$ & $\begin{array}{l}\text { Importância unânime } \\
\text { de simulações no } \\
\text { laboratório. }\end{array}$ & $\begin{array}{l}\text { Laboratório oferece } \\
\text { segurança para cuidado } \\
\text { aos pacientes. }\end{array}$ \\
\hline $\begin{array}{l}\text { Coutinho e } \\
\text { Friedlander } \\
(2004)\end{array}$ & $\begin{array}{l}\text { Avaliar desempenho } \\
\text { psicomotor no LE* e } \\
\text { CC }^{* *}\end{array}$ & 77 estudantes & $\begin{array}{l}\text { Quantitativo. } \\
\text { Instrumento para } \\
\text { coleta de dados. }\end{array}$ & $\begin{array}{l}\text { Valores sem } \\
\text { correlação } \\
\text { significativa. }\end{array}$ & $\begin{array}{l}\text { Melhor desempenho } \\
\text { psicomotor no CC. }\end{array}$ \\
\hline
\end{tabular}

*LE: laboratório de enfermagem; ${ }^{*}$ CC: centro cirúrgico.

ausculta respiratória, e, ainda, a simulação de terapia intensiva, que envolveu programadores de sistemas, professores e acadêmicos.

Em relação ao delineamento dos estudos, um artigo combinou o método quantitativo e qualitativo, e seis são quantitativos de corte transversal. Um trabalho aplicou a abordagem qualitativa, e outro 
caracterizou-se como relato de experiência. Nenhum estudo foi classificado como ensaio clínico aleatorizado. Isso significa que não foi encontrada evidência científica de que o uso da simulação para o ensino de técnicas e procedimentos exerce efeito positivo sobre o desempenho clínico dos estudantes de graduação em enfermagem.

Na categoria Simulação por Computador, emergiram sete artigos com temas relacionados à propedêutica e aos procedimentos e técnicas que, se não forem realizados corretamente, apresentam risco para os pacientes. O pressuposto desses estudos é que o uso da tecnologia nas simulações reforça a responsabilidade do aluno referente ao desempenho próprio. Em alusão ao desafio real, o processo estimula a aprendizagem e, assim, as simulações são oportunidades para os estudantes entenderem as consequências de suas ações em um cenário virtual, que possibilita o desenvolvimento de competências reais (Sasso, Souza, 2006).

Objetos educacionais digitais são ferramentas de apoio ao ensino presencial de Fundamentos de Enfermagem (Schatkoski et al., 2007). Na propedêutica de ausculta respiratória, os alunos de Semiologia têm expressado dificuldades para reconhecer os sons respiratórios, identificar as áreas de ausculta e, ainda, distinguir os sons normais e os adventícios (Melo, Damasceno, 2006). Considerando que os métodos convencionais para o ensino da ausculta dos sons respiratórios não têm sido eficientes, Melo e Damasceno (2006) desenvolveram um software como recurso na aprendizagem dessa prática. A ferramenta compreendeu: o método da ausculta, as finalidades do procedimento, a classificação dos sons e as características estetoacústicas. Os recursos tridimensionais incluíram a construção de avatares e de ambientes virtuais, e a conclusão do trabalho foi que a simulação da ausculta respiratória, proporcionada por esse software, contribuiu para o ensino e a aprendizagem em enfermagem (Melo, Damasceno, 2006).

Buscando aprimorar o ensino da administração de oxigênio aos pacientes, docentes e alunos da Universidade Federal do Rio Grande do Sul (UFRGS) desenvolveram e avaliaram o uso de objetos digitais para o treino desse procedimento, nas formas de hipertexto, jogo educativo e simulação da passagem do cateter (Schatkoski et al., 2007). Foi aplicado um questionário para 44 acadêmicos da disciplina Fundamentos do cuidado humano III, a fim de se verificar a adequação do conteúdo, apresentação visual e utilização desses objetos. As dificuldades referiram-se à visualização e à maneira de salvar o material digital; mas os resultados sugeriram que esses recursos reforçaram a participação ativa do estudante na aprendizagem. Os acadêmicos tiveram boa receptividade ao hipertexto, ao jogo educativo e à simulação sobre oxigenoterapia (Schatkoski et al., 2007).

A utilização dos mesmos objetos para o treino de oxigenoterapia foi investigada também por dez docentes do curso de graduação em Enfermagem da UFRGS (Cogo et al., 2009). Foi aplicada uma escala do tipo Likert para avaliar o conteúdo, a apresentação visual e a utilização do material digital. A interatividade do estudante na aprendizagem ficou evidente com a avaliação positiva que os docentes fizeram da simulação e do jogo educativo para o ensino da oxigenoterapia (Cogo et al,. 2009).

Estudar com o apoio de simulações no computador, antecipando procedimentos que serão realizados em manequins e, posteriormente em pacientes, é uma estratégia na qual o acadêmico assume uma postura ativa na aprendizagem (Schatkoski et al., 2007). Para favorecer as habilidades psicomotoras, os professores de disciplinas que iniciam o estudante na clínica precisam desenvolver sensibilidade para entender as dificuldades e os sentimentos envolvidos na execução dos primeiros procedimentos (Gomes, Germano, 2007).

No ambiente clínico, os professores selecionam os pacientes, iniciando com uma abordagem de controle sobre as ações do estudante, sendo essa dependente da experiência e competência do mesmo. O continuum de aprendizagem das habilidades técnicas e o desenvolvimento da confiança do acadêmico no cuidado aos pacientes pode começar com procedimentos simulados, sob orientações que deverão ser gradualmente reduzidas, culminando na prática supervisionada nas situações reais. Nesse processo, o professor deve oferecer orientações prévias e posteriores ao atendimento, estando disponível para as dúvidas e, ainda, avaliando o desempenho do aluno.

Silva e Cogo (2007) analisaram o desempenho de 37 acadêmicos de enfermagem da UFRGS, na realização da punção venosa, que foi ensinada com o recurso de objetos educacionais digitais. A coleta dos dados foi feita por observação direta durante a prática no manequim, e, na sequência, os estudantes 
preencheram um questionário sobre a utilização dos objetos educacionais digitais, que avaliou dez passos do procedimento no laboratório: preparação do material; lavagem das mãos; uso das luvas; procurar a veia; "garrotear" o membro; assepsia; introdução do cateter de plástico sobre agulha e retirada do garrote ao retorno do sangue; pressionar o cateter ao retirar o mandril; demonstrar a técnica de fixação do cateter; demonstrar conhecimento sobre anticoagulação do acesso ou proceder à infusão intravenosa. Dentre os estudantes que não tinham experiência prévia em punção venosa, $72,97 \%$ realizaram corretamente $50 \%$ do procedimento. Os autores entenderam que a prática no laboratório permite erros até que se alcance o acerto, gerando segurança no aluno (Silva, Cogo, 2007).

Os jogos educativos e a simulação digital contribuem para que o conhecimento, adquirido em experiências virtuais, seja aplicado nas situações reais. A aprendizagem torna-se significativa quando o processo de ensino é organizado e intencional, requerendo a predisposição de educação contínua da parte dos professores (Karino, Guarient, 2001). Nessa trajetória, destaca-se a necessidade de preparação dos docentes que atuam no desenvolvimento das habilidades clínicas dos estudantes de enfermagem. A integração da teoria à prática, sob a liderança de professores com competência clínica e didática, contribui para que os acadêmicos desenvolvam segurança crescente, iniciando-se com simulação de procedimentos, seguindo para os cuidados aos pacientes durante as aulas práticas e se estendendo às atividades posteriores de estágio.

Após o ensino de procedimentos, como a realização de punção venosa, a aprendizagem expande-se para ambientes assistenciais que requerem o domínio de alta tecnologia. Os cuidados intensivos com os pacientes representam desafios para os acadêmicos, que precisam aprender, também, sobre a monitorização dos equipamentos que traduzem os parâmetros clínicos. Sob essa perspectiva, os estudantes têm a responsabilidade de prestar cuidados conforme a demanda de assistência, exercitando o conhecimento previamente adquirido (Barbosa, Marin, 2000).

O estudo de Barbosa e Marin (2000), realizado na Universidade Federal de Santa Catarina (UFSC), sobre desenvolvimento e aplicação de um ambiente web de simulação clínica em terapia intensiva, apresentou dados preliminares de um paciente com lesão por arma branca em hemitórax direito, que foi admitido na unidade de terapia intensiva, evoluindo para insuficiência respiratória, com necessidade de ventilação mecânica. Os alunos do $6^{\circ}$ período, que cursavam a disciplina Enfermagem nas intercorrências cirúrgicas e de urgência, selecionaram os dados do paciente para propor as ações de enfermagem, observando os seguintes temas: avaliação do indivíduo em estado crítico, insuficiência respiratória, ventilação mecânica e drenagem torácica.

Os resultados do uso da web demonstraram boa aceitação da simulação pelos alunos, com destaque para o estímulo ao aprendizado, retenção do conteúdo e satisfação na utilização do ambiente virtual (Barbosa, Marin, 2000). O estudo ofereceu subsídios para o ensino em terapia intensiva, estimulando o pensamento crítico do estudante e o questionamento da prática. Barbosa e Marin (2000) mostraram a viabilidade de simulação na web direcionada para a educação, contribuindo para a construção de tecnologias educacionais para os cursos de graduação de Enfermagem.

A simulação computadorizada reduz a distância entre a teoria e a prática, pois o computador apresenta uma situação parecida com a realidade (Sasso, Souza, 2006). Sasso e Souza (2006) descreveram a construção de um ambiente computacional para uma situação simulada de parada cardiorrespiratória, demonstrando que o software reduz o estresse para o aluno e o risco para o paciente. A experiência sugeriu que a simulação pode evitar que o paciente sofra danos decorrentes de falhas do estudante no processo decisório, contribuindo para a assistência adequada em situação real.

Para conhecer a opinião de discentes do $8^{\circ}$ período de Enfermagem, sobre a utilização dos computadores em simulações, Telles Filho e Cassiani (1999) desenvolveram escalas de Likert com itens favoráveis e desfavoráveis ao objeto de estudo, cujas respostas indicaram o nível de concordância, de indecisão ou de discordância dos estudantes, em relação às afirmações específicas. Essas afirmações foram elaboradas a partir da reunião e da seleção de frases fundamentadas na literatura, com pertinência à temática, e advindas da experiência dos investigadores e do estudo-piloto. Os resultados demonstraram que $58,4 \%$ dos 101 estudantes consideraram o computador como um recurso importante para o ensino, sendo receptivos à utilização da simulação computadorizada e à atualização de técnicas pedagógicas, com interesse na interatividade (Telles Filho, Cassiani, 1999). 
Os jogos educativos e as simulações virtuais permitem que os estudantes desenvolvam habilidades para a realização de procedimentos, observando as técnicas. Os jogos online informam o desempenho; a simulação computadorizada reduz o medo de errar, aceitando as tentativas do estudante para a resolução do problema (Schatkoski et al., 2007). Embora existam vantagens na utilização de computadores para o ensino de avaliações, procedimentos e técnicas de enfermagem, há dificuldade na transferência do conteúdo das simulações para as situações reais. O teclado e o mouse do computador compõem a interface entre o estudante e o paciente virtual, uma situação que não ocorre na realidade, determinando que a prática clínica é essencial para promover a sensibilidade na assistência (Gomes, Germano, 2007; Sasso, Souza, 2006).

Um fator importante sobre as simulações com computadores é a presença do professor, pois a utilização exclusiva da tecnologia não é suficiente (Sasso, Souza, 2006). A presença do docente é necessária, sendo a informática um dispositivo integrante do ensino da terapia intravenosa (Dias et al., 2005). Portanto, as tecnologias digitais oferecem suporte; mas o professor deve incentivar os acadêmicos na busca de conhecimento técnico e humano, tornando-se o elo entre as relações pessoais e o desempenho para facilitar o processo de enfermagem (Karino, Guarient, 2001).

Para os estudantes que necessitam desenvolver habilidades psicomotoras, as atividades de simulação realizadas no laboratório são complementares à aprendizagem de procedimentos. Na segunda categoria, Laboratório de Enfermagem, os dois artigos selecionados acentuam a importância desse ambiente na formação do enfermeiro (Gomes, Germano, 2007; Coutinho, Friedlander, 2004). A noção subjacente a esses estudos é a de que as simulações contribuem para que os estudantes desenvolvam as habilidades necessárias aos cuidados, em situações anteriores aos atendimentos na clínica. Nas primeiras experiências com os pacientes, os estudantes sentem ansiedade, que é expressa por tremores, palidez e sudorese. Esses sintomas podem estar associados ao desmaio, choro e descontrole emocional. Quando é assegurado o tempo adequado para a aprendizagem no laboratório, a ansiedade na área clínica é atenuada (Gomes, Germano, 2007).

As opiniões dos estudantes sobre o desenvolvimento de habilidades reforçam as contribuições das simulações no laboratório, consideradas recursos que facilitam o ensinar e o aprender, particularmente na execução de técnicas (Gomes, Germano, 2007).

Para demonstrar a importância do laboratório na aprendizagem de procedimentos, Gomes e Germano (2007) classificaram as informações geradas por grupos focais de estudantes da Universidade Federal do Rio Grande do Norte (UFRGN), em três categorias: empatia que deve ser desenvolvida durante os cuidados, insegurança inicial do aluno e papel do professor. Segundo esses autores, a simulação dos procedimentos no laboratório prepara tecnicamente o estudante para oferecer cuidado com qualidade aos pacientes (Gomes, Germano, 2007).

Os erros com os pacientes não podem ser tolerados, sendo fundamental a segurança para a aprendizagem dos procedimentos (Coutinho, Friedlander, 2004). No entanto, nas simulações, os acadêmicos podem errar para aprimorar as técnicas, executando os procedimentos sem temerem os danos, pois as atividades em laboratório facilitam a transição para a realidade assistencial (Silva, Cogo, 2007). Coutinho e Friedlander (2004) avaliaram as habilidades psicomotoras de 77 alunos da disciplina Enfermagem em centro cirúrgico, comparando o manuseio de material esterilizado no laboratório de enfermagem e na sala operatória. Considerando o número de erros, o desempenho motor dos acadêmicos foi melhor no centro cirúrgico; porém, a diferença nas habilidades psicomotoras nos dois ambientes não foi estatisticamente significativa. Os dois fatores que podem ter contribuído para a redução do número de erros no centro cirúrgico foram: o retorno dos estudantes ao laboratório para o treino de habilidades e o conhecimento prévio em manuseio de material esterilizado (Coutinho, Friedlander, 2004).

Como recurso educacional, o laboratório de enfermagem deve ser disponibilizado ao estudante para a prática dos procedimentos, em uma transição entre a teoria e a clínica. Esse ambiente, no entanto, não se restringe às técnicas, mas proporciona uma relação entre o professor e o estudante, gerando conhecimento e reflexão (Gomes, Germano, 2007). A humanização do cuidado, essencial na prática dos estudantes de enfermagem, é desenvolvida exclusivamente nas situações reais. Porém, as inovações tecnológicas aplicadas aos simuladores têm gerado programas computacionais, modelos e manequins, 
que buscam uma aproximação com a anatomia, a fisiologia e as respostas ao processo saúde-doença dos seres humanos.

\section{Considerações finais}

A simulação é uma estratégia utilizada nas disciplinas de graduação do curso de Enfermagem para o ensino de técnicas e procedimentos necessários para a realização de cuidados. No Brasil, as experiências indicam que as condições simuladas têm contribuido para a prática dos estudantes, na transição de ambientes virtuais e controlados em laboratórios para a assistência aos pacientes.

Os resultados desta revisão sugerem que a aplicação de estratégias educacionais no laboratório pode ser associada à tecnologia computacional, como apoio ao ensino presencial. Há disposição dos docentes e discentes para formas inovadoras no ensino, particularmente as simulações; porém, dos nove estudos selecionados nesta revisão, nenhum caracterizou-se como ensaio clínico aleatorizado, "padrão ouro" de evidências científicas. Essa informação demonstra a necessidade de realização de pesquisas nessa área, cujos resultados indicarão qual é a relação entre as simulações para o ensino de técnicas e procedimentos de enfermagem e o desempenho dos estudantes na clínica.

\section{Colaboradores}

Ilka Nicéia D'Aquino Oliveira Teixeira responsabilizou-se pela concepção e planejamento do estudo, obtenção, análise e interpretação dos dados e redação do trabalho. Jorge Vinícius Cestari Félix colaborou na redação do texto e responsabilizouse pela revisão crítica técnica do trabalho.

\section{Referências}

BARBOSA, S.F.; MARIN, H.F. Web based simulation: a tool for teaching critical care nursing. Rev. Latino-am. Enferm., v.17, n.1, p.7-13, 2000.

BARDIN, L. Análise de conteúdo. 3.ed. Lisboa: Edições 70, 2004.

BECK, D.; SRIVASTAVA, R. Perceived level and source of stress in baccalaureate nursing students. J. Nurs. Educ., v.30, n.3, p.127-32, 1999.

BECKMAN, T.J.; LEE, M.C. Proposal for a collaborative approach to clinical teaching. Mayo Clin. Proc., v.84, n.4, p.339-44, 2009.

CAREGNATO, R.C.; MUTTI, R. Pesquisa qualitativa: análise de discurso versus análise de conteúdo. Texto Contexto Enferm., v.15, n.4, p.679-84, 2006.

COGO, A.L. et al. Objetos educacionais digitais em enfermagem: avaliação por docentes de um curso de graduação. Rev. Esc. Enferm. USP, v.43, n.2, p.295-9, 2009.

COUTINHO, R.M.; FRIEDLANDER, M.R. Manuseio de material esterilizado: processo ensino-aprendizagem em laboratório de enfermagem e em centro cirúrgico. Acta Paul. Enferm., v.17, n.4, p.419-24, 2004.

DIAS, D.C. et al. Terapia intravenosa na web: um recurso didático. Cogitare Enferm., v.10, n.3, p.23-7, 2005.

GOMES, C.O.; GERMANO, R.M. Processo ensino/aprendizagem no laboratório de enfermagem: visão de estudantes. Rev. Gaucha Enferm., v.28, n.3, p.401-8, 2007. 
HART, G.; ROTEM, A. The best and the worst: students' experience of clinical education. Austr. J. Adv. Nurs., v.11, n.3, p.26-33, 1994.

JEFFRIES, P.R. Clinical simulations in nursing education: valuing and adopting an experiential clinical model. Create Future, v.4, n.7, p.2-3, 2007. Disponível em: <http://www.nursingsociety.org/Publications/Newsletter/Documents/ CTF_V4_7.pdf>. Acesso em: 20 jul. 2009.

A framework for designing, implementing, and evaluating simulations used as

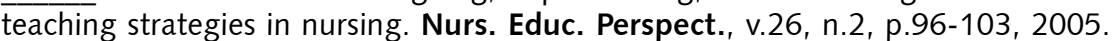

KARINO, M.E.; GUARIENT, M.H. O aprendizado no primeiro estágio da enfermagem: a visão do aluno. Arq. Cienc. Saude Unipar, v.5, n.1, p.33-9, 2001.

MEDLEY, C.F; HORNE, C. Using simulation technology for undergraduate nursing education. J. Nurs. Educ., v.44, n.1, p.31-4, 2005.

MELO, F.N.; DAMASCENO, M.M. A construção de um software educativo sobre ausculta dos sons respiratórios. Rev. Esc. Enferm. USP, v.40, n.4, p.563-9, 2006.

NEHRING, W.M.; LASHLEY, F.R. Current use and opinions regarding human patient simulators in nursing education: an international survey. Nurs. Educ. Perspect., v.25, n.5, p.244-8, 2004.

ROTHGEB, M.K. Creating a nursing simulation laboratory: a literature review. J. Nurs. Educ., v.47, n.11, p.489-94, 2008.

SASSO, G.T.; SOUZA, M.L. A simulação assistida por computador: a convergência no processo de educar-cuidar da enfermagem. Texto Contexto Enferm., v.15, n.2, p.231-9, 2006.

SCHATKOSKI, A.M. et al. Hipertexto, jogo educativo e simulação sobre oxigenoterapia: avaliando sua utilização junto a acadêmicos de enfermagem. Online Braz. J. Nurs., v.6, 2007. Disponível em: <http://www.uff.br/objnursing/index.php/ nursing/article/ view/636/149>. Acesso em: 12 dez. 2009.

SEROPIAN, M.A. et al. Simulation: not just a manikin. J. Nurs. Educ., v.43, n.4, p.164-9, 2004.

SHARIF, F.; MASOUMI, S. A qualitative study of nursing student experiences of clinical practice. BMC Nurs., v.4, n.6, p.1-7, 2005. Disponível em: <http:// www.biomedcentral.com/1472-6955/4/6>. Acesso em: 25 fev. 2010.

SILVA, A.P.; COGO, A.L. Aprendizagem de punção venosa com objeto educacional digital no Curso de Graduação em Enfermagem. Rev. Gaucha Enferm., v.28, n.2, p.87-92, 2007.

TELLES FILHO, P.C.; CASSIANI, S.H. O computador no ensino de enfermagem: análise das atitudes de discentes de instituições de nível superior. Rev. Latino-am. Enferm., v.7, n.1, p.93-8, 1999.

TUORINIEMI, P.; SCHOTT-BAER, D. Implementing a highfidelity simulation program in a community college setting. Nurs. Educ. Perspect., v.29, n.2, p.105-9, 2008. 
TEIXEIRA, I.N.D.O.; FELIX, J.V.C. Simulación como estrategia de enseñanza de enfermería: revisión de literatura. Interface - Comunic., Saude, Educ., v.15, n.39, p.1173-83, out./dez. 2011.

El objetivo de esta revisión es presentar la simulación como estrategia de enseñanza para estudiantes de graduación en Enfermería, según estudios hechos en Brasil. La búsqueda fue realizada en las bases de datos BDENF, Lilacs e Medline, utilizándose de las palabras clave: enseñanza, educación, laboratório, simulación y enfermería. Fueron inclusos los artículos científicos sobre simulación como estrategia de enseñanza, publicados en lengua portuguesa, de enero de 1999 a septiembre de 2010. Para análisis de datos, fue aplicada la tecnica de análisis de contenido, descrita por Bardin. Nueve artículos fueron selecionados, siendo clasificados en dos categorias: siete en "simulación por computador" y dos en "laboratório de enfermería". Los resultados indican que la simulación contribuye para la adquisición de habilidades psico-motoras y auto-confianza. No fueron encontrados ensayos clínicos a respeto de la eficiencia de la simulación en la práctica de los estudiantes de enfermaría. Se necesitan más pesquisas sobre el tema.

Palabras clave: Instrucción por computador. Estudiantes de Enfermería. Simulación. 